\title{
INFLUENCIA DEL SISTEMA DE EVALUACIÓN EMPLEADO EN LA PERCEPCIÓN DEL ALUMNO SOBRE SU APRENDIZAJE Y LAS COMPETENCIAS DOCENTES (1)
}

Influence of the evaluation system on the students learning perception and the teaching skills

Influenciar o sistema de avaliação usado em percepções de suas habilidades de ensino e aprendizagem dos alunos

\author{
David Hortigüela Alcalá (2) \\ Víctor Abella García (3) \\ Vanesa Delgado Benito (4) \\ Vanesa Ausín Villaverde (5)
}

\begin{abstract}
(1) Este trabajo ha sido apoyado por el Programa de Estado de Investigación, Desarrollo e Innovación frente a los desafíos de la Sociedad, 2013 a 2016. (Ministerio de Economía y Competitividad - España) [subvención número Ref .: EDU 2013-42024-R].

(2) Universidad de Burgos, España. Facultad de Educación. Fono: +34 947259517. Correo electrónico: dhortiguela@ubu.es

(3) Universidad de Burgos, España. Facultad de Educación. Fono: +34 947258799. Correo electrónico: vabella@ubu.es

(4) Universidad de Burgos, España. Facultad de Educación. Fono: +34 947258723. Correo electrónico: vdelgado@ubu.es

(5) Universidad de Burgos, España. Facultad de Educación. Fono: +34 947259317. Correo electrónico: vausin@ubu.es
\end{abstract}

\section{Resumen}

El presente estudio, realizado en la Universidad de Burgos (España), analiza la percepción del alumnado universitario acerca del aprendizaje y las competencias docentes. Participaron 485 alumnos de cinco asignaturas de dos grados: maestro en Educación Primaria y maestro de Educación Infantil. Estos se dividen en dos grupos, (A el de primaria y B el de infantil), impartido cada uno por dos profesores. En el grupo de Primaria se ha utilizado un sistema de evaluación formativa, basado en autoevaluaciones grupales y coevaluaciones intra e intergrupales a lo largo del proceso. Sin embargo, en el grupo B el sistema de evaluación ha sido más tradicional, asociándose a la calificación final de un examen y a la realización de trabajos. La metodología del trabajo ha sido mixta, empleándose tanto un análisis descriptivo (medias y desviación típica por factores) e inferencial (tablas de contingencia y Influencia del sistema de evaluación empleado en la percepción del alumno sobre su aprendizaje y las competencias docentes 
correlaciones de Pearson y ANOVAS) como cualitativo (entrevistas a los dos docentes). Se utiliza un diseño pretest-postest, comprobando la variabilidad de la percepción al finalizar las asignaturas. Se emplean dos cuestionarios, la escala de formación inicial del profesorado y el cuestionario de evaluación de competencias docentes. Se observa cómo el alumnado que participó en el sistema de evaluación formativa (grupo A) tiene una percepción más elevada tanto en el aprendizaje como en las competencias docentes, estableciéndose diferencias significativas en este factor. En la parte cualitativa se manifiesta la disparidad de percepciones entre docentes respecto al rol que ha de desempeñar el alumno como agente del proceso de aprendizaje.

Palabras clave: evaluación auténtica, evaluación para el desempeño, aprendizaje, competencias docentes, metodología mixta.

\begin{abstract}
This study, conducted at the University of Burgos (Spain), analyzes the perception of university students about learning and skills developed by the teacher. Participants were 485 students from five subjects of two degrees: Primary Teacher Education and Early Childhood teacher. These are divided into two groups each taught by two teachers. In the Primary group it used a formative assessment system based on self-assessment group and intra and inter-process along peer assessment. However, in the two subjects in group B the evaluation system used was more traditional, associating the final qualifying examination and the conduct of work demanded by the teacher. The methodology of work has been mixed, using both a descriptive analysis (mean and standard deviation factors) and inferential (contingency tables and Pearson correlations and ANOVA) and qualitative (interviews with the two teachers). A pretest-posttest design is used, checking the variability of perception at the end of the courses. Two questionnaires, the scale of initial teacher training and assessment questionnaire used teaching skills. It is observed how the students who participated in the formative assessment system (group A) has a higher perception both learning and teaching skills, establishing significant differences in this factor. In the qualitative part the disparity in perceptions among teachers regarding the role to be played by the student as an agent of the learning process.
\end{abstract}


Keywords: authentic assessment, performance based assessment, learning, teaching skills, mixed methods research.

\section{Resumo}

Este estudo, realizado na Universidade de Burgos (Espanha), analisa a percepção de estudantes universitários sobre as habilidades de aprendizagem e ensino. 485 alunos participaram em cinco cursos de dois graus: Mestrado em Ensino Fundamental e professor da Primeira Infância. Estes são divididos em dois grupos (A e B sobre a criança primário), cada ensinado por dois professores. No grupo primário é utilizado um sistema de avaliação formativa baseada em grupo de auto-avaliação e intra e interprocesso junto coevaluaciones. No entanto, nos dois sujeitos no grupo B o sistema de avaliação dos funcionários era mais tradicional, associando o exame de qualificação final e a conduta de trabalho exigido pelo professor. A metodologia de trabalho tem sido misturados, usando tanto uma análise descritiva (média e fatores de desvio padrão) e inferencial (tabelas de contingência e correlações de Pearson e ANOVA) e qualitativos (entrevistas com os dois professores). Um desenho pré-teste-pós-teste é utilizada, verificar a variabilidade de percepção no final dos cursos. Dois questionários, a escala da formação inicial de professores e ensino de habilidades questionário de avaliação utilizados. Ele mostra como os alunos que participaram no sistema de avaliação formativa (grupo A) tem uma maior percepção de aprendizagem e habilidades de ensino, estabelecendo diferenças significativas neste fator. $\mathrm{Na}$ parte qualitativa da disparidade de percepções entre os professores sobre o papel a ser desempenhado pelo aluno como agente do processo de aprendizagem ocorre.

Palavras-chave: avaliação autêntica, avaliação de desempenho, de aprendizagem, habilidades de ensino, metodologia mista .

\section{Introducción}

En la última década se han experimentado cambios sustanciales en el paradigma socioeducativo, afectando directamente tanto a las modalidades de aprendizaje como a la configuración de los estudios universitarios (Johnson, 2014). La formación del estudiante ha de estructurarse bajo unos principios metodológicos que favorezcan la autonomía en su aprendizaje, la toma de decisiones en la regulación del trabajo y su 
implicación de manera activa en su formación (Hidalgo, Bordallo, Sánchez y Cantabrana, 2012). Este cambio en el modelo instruccional se ve claramente influido por la disminución de la duración de las asignaturas, el aumento en la impartición de las titulaciones semipresenciales y online, así como por el uso de metodologías de carácter innovador que dejan en un segundo plano técnicas de carácter más tradicional como las clases magistrales (Wold, 2013). Del mismo modo, el acceso al mundo laboral del alumnado universitario requiere de la adquisición de destrezas no solamente vinculadas al aprendizaje instrumental, ya que el ámbito relacional y organizativo se convierten en aspectos clave para el desempeño diario de cualquier actividad (Chan, 2015). Este hecho cobra una especial relevancia en la formación inicial del profesorado, ya que si el futuro maestro ha recibido un aprendizaje estructurado a partir del trabajo de las emociones y de la educación en valores repercutirá en una enseñanza de más calidad y más comprometida e igualitaria (Peiró, 2012).

Es por ello que la metodología utilizada por el docente y el sistema de evaluación asociado tienen una incidencia directa, ya no solo en el aprendizaje del alumno, sino en su motivación, implicación en las tareas y en la competencia percibida (Hortigüela, Pérez-Pueyo y López-Pastor, 2015). Se trata de que el alumno se sienta partícipe de aquello que aprende, tenga diferentes alternativas para resolver los problemas que se le plantean y que, además, pueda decidir cómo hacerlo. No tiene sentido formar a un futuro maestro a partir de la memorización de textos o realización de trabajos bajo guiones estructuradamente cerrados, ya que en su futura práctica profesional (sea en infantil o en primaria) deberá fomentar la reflexión comprensiva en cada una de las situaciones de aprendizaje (Dekker-Groen, Van der Schaaf y Stokking, 2013). Por lo tanto, hemos de entender la evaluación como ese proceso sistemático orientado a unos fines claramente formativos y no enfocados únicamente a una calificación final, permitiendo al alumno conocer realmente el trabajo que lleva realizado y asesorarle sobre aquello que tiene que hacer para mejorarlo (Canabal y Castro, 2012). La aplicación de estos sistemas de manera regulada y estructurada permite que ese aprendizaje adquirido sea más transferible a otros contextos, independientemente de que estén o no vinculados a los conceptos específicos adquiridos (Gikandi, 2013). 
Independientemente del sistema metodológico y evaluativo empleado, es fundamental valorar su idoneidad mediante la valoración que realiza de los mismos el principal agente de intervención, el alumno. Solamente si damos voz al estudiante sobre su proceso formativo y valoramos su percepción objetivamente, nos daremos cuenta del grado de cumplimiento de las pretensiones y los objetivos iniciales planteados (Varsavsky, Matthews y Hodgson, 2014). Esta percepción puede atender a diferentes aspectos, incidiendo en las competencias desarrolladas por el docente en las asignaturas impartidas. Además puede estar influida por diferentes variables, relacionadas tanto con sus experiencias previas como por factores internos y personales. En la presente investigación se atiende a este hecho, en concreto a las variables independientes de experiencias previas en evaluación formativa, edad y expediente académico.

Algunas de las limitaciones que se observan en investigaciones fundamentadas en la percepción de alumno se encuentran en la falta de contraste de las mismas con la valoración que pueda tener el docente sobre el proceso llevado a cabo. Esto puede conllevar el desarrollo de un enfoque únicamente unidireccional, perdiendo la perspectiva global que otorga valorar a todos los agentes participantes de la investigación (Serván, 2011). Para ello es idóneo utilizar técnicas de investigación variadas que permitan extraer la máxima información a lo largo del proceso, valorando el trabajo realizado en el aula, la percepción del alumno sobre lo acontecido y la valoración del docente acerca del planteamiento metodológico desarrollado. Autores como Heyvaert, Hannes, Maes y Onghena (2013) recomiendan el empleo de metodologías mixtas, combinando técnicas cuantitativas y cualitativas que den respuesta a los objetivos del trabajo.

Por lo tanto, en la presente investigación, y bajo el empleo de una metodología mixta, se analiza la percepción que tiene el alumno acerca del aprendizaje adquirido y de las competencias desarrolladas por el docente, utilizando en cada grupo un sistema de evaluación diferente. Esto se contrasta con la realización de entrevistas a los docentes de cada uno de los grupos, incidiendo en la concepción de la enseñanza a partir de la metodología utilizada. Por ello, en esta investigación se profundiza en una temática relevante en el panorama educativo actual, arrojando nuevos datos de variables a los ya 
existentes en la bibliografía y estableciendo una aplicabilidad en futuras líneas de investigación.

\section{Objetivos}

- Comparar la percepción que tiene el alumnado sobre el aprendizaje obtenido y las competencias del docente en función del sistema de evaluación empleado.

- Analizar la transferencia del aprendizaje en cada uno de los grupos en función de la edad, la experiencia previa en sistemas de evaluación formativa y el expediente académico.

- Contrastar las valoraciones de los docentes que han impartido cada uno de los grupos acerca de la relevancia que tiene el tipo de evaluación empleado en el aprendizaje del alumno.

\section{Método}

\subsection{Participantes}

Han participado 485 estudiantes (61,2\% mujeres y 38,8\% hombres) con una media de edad de 21.29 años (DT = 1.89) matriculados en dos grados de la Facultad de Educación de la Universidad de Burgos y de León: Maestro en Educación Primaria y Maestro en Educación Infantil. Los alumnos forman parte de cinco asignaturas; Educación Física y su Didáctica e Investigación Educativa del Grado en Educación Primaria, y Desarrollo Psicomotor I, Desarrollo Psicomotor II y Habilidades Perceptivo-Motrices y el Juego Infantil del Grado en Educación Infantil.

Se han realizado dos grupos, en el que cada uno ha recibido un sistema de evaluación diferente. El grupo A, compuesto por las dos asignaturas de Primaria (235 alumnos) que participó en un proceso de evaluación formativa. El grupo B, integrado por 250 alumnos matriculados en las tres asignaturas de Infantil, cuyo sistema de evaluación se articuló a partir de calificación de exámenes y trabajos finales. Tras realizar la prueba de SHAPIRO-WILK se observa que la muestra responde a parámetros de normalidad $(p=.131)$. Las materias de cada grupo han sido impartidas por dos docentes diferentes. Los dos docentes del grupo A tienen una experiencia en la docencia 
universitaria de cinco y 15 años respectivamente, mientras que los dos del grupo B de 13 y 22.

En la Tabla ,1 se pueden observar los dos grupos generados, el tipo de evaluación que han recibido y el número de alumnos y profesores que integran cada uno.

Tabla 1.

Grupos generados en el estudio, asignaturas incluidas y alumnos y profesores participantes en cada sistema de evaluación.

\begin{tabular}{llcccc}
\hline Titulación & Asignatura/s & Grupo & Evaluación recibida & Profesores & Alumnos \\
\hline $\begin{array}{l}\text { Grado } \\
\text { Primaria }\end{array}$ & $\begin{array}{l}\text { EF y su Didáctica (Burgos) } \\
\text { Investigac. Educativa (León) }\end{array}$ & A & Evaluación formativa & 2 & 235 \\
\hline \multirow{2}{*}{$\begin{array}{l}\text { Grado } \\
\text { Infantil }\end{array}$} & $\begin{array}{l}\text { Dllo. Psicomotor I (Burgos) } \\
\text { Dllo Psicomotor II (Burgos) }\end{array}$ & \multirow{2}{*}{$\begin{array}{l}\text { B } \\
\text { Hab. Perceptivo-Motrices y } \\
\text { el Juego Infantil (León) }\end{array}$} & Evaluación calificadora & \multirow{2}{*}{$2^{*}$} & 250 \\
\hline
\end{tabular}

*El profesor de las asignaturas de Dllo. Psicomotor I y II es el mismo.

\subsection{Instrumentos}

\subsubsection{Cuantitativos}

Se han empleado dos cuestionarios para la recogida de datos. El primero de ellos es la Escala de Formación Inicial del Profesorado (Castejón, Santos y Palacios, 2015). Esta escala valora la percepción del alumnado sobre la evaluación recibida, su implicación en el proceso y el uso del feedback para la mejora del proceso formativo. Tiene 17 cuestiones a las que los estudiantes responden según su grado de acuerdo o desacuerdo con el enunciado en una escala tipo Likert, cuyos valores van desde 1 (Nada) hasta 5 (Mucho). Tiene una fiabilidad según el alfa de Cronbach de 0.842, superior al límite inferior que según Corbetta (2007) es aceptado como fiable.

El segundo cuestionario es el de evaluación de Intervención Docente (CEID) validado por Moreno-Murcia, Silveira y Belando (2015). Evalúa las competencias docentes en el ámbito universitario, incidiendo en la metodología utilizada, la organización de las clases, la interacción con el estudiante y la flexibilidad y dinamismo en el programa desarrollado. Tiene 28 cuestiones a las que los estudiantes responden en una escala Likert, donde el 1 es totalmente en desacuerdo y el 5 totalmente de acuerdo. Tiene una fiabilidad según el alfa de Cronbach de 0.831 . 
Con respecto a la validez, la prueba piloto realizada a los cuestionarios confirma el significado previsto de las variables que se pretenden medir, comprobando la utilidad práctica de los instrumentos. A partir de la identificación de las variables predictivas de los cuestionarios se definieron operacionalmente cada uno de los ítems de los que constan los dos instrumentos, relacionados estrechamente con los objetivos de la presente investigación.

Para los dos cuestionarios se realizó un Análisis Factorial de Componentes Principales, con el fin de evaluar la bondad del ajuste de los datos. Se obtienen valores adecuados para el índice KMO de 0.813 como en el test de esfericidad de Barlett ( $\mathrm{p}>$ $.00)$.

El Análisis Factorial Exploratorio ha revelado una solución de dos factores con un autovalor superior a uno. Estos dos factores explican el total de la varianza, una vez corregida y rotada la matriz de componentes (Varimax Normalizada). El autovalor inicial para el primer factor corresponde a $58.634 \%$ de la varianza, mientras que el segundo al $41.366 \%$ de la misma.

Los índices obtenidos en la matriz de covarianzas presentaron ajustes satisfactorios para el índice RMSEA (Root Mean Square Error Aproximation) $=0.063$. En este índice los valores inferiores a .05 indican un buen ajuste, y valores de hasta .08 representan errores razonables de aproximación.

En el CFI (Comparative Fit Index ) y GFI (Goodness of Fit Index), se alcanza un valor de 0.9, y 0.92 indicativo de buen ajuste.

Son dos los factores resultantes del análisis factorial realizado a los cuestionarios y en los que se agrupan la totalidad de los ítems que lo componen:

a)Aprendizaje obtenido en el sistema de evaluación (12 ítems): se establecen cuestiones relacionadas con la regulación de las tareas, la interacción entre profesor y alumno, la implicación del alumno en el trabajo, la toma de decisiones y la autonomía en la elaboración de las actividades.

b)Competencias docentes empleadas en el sistema (16 ítems): se incluyen ítems vinculados a la planificación del proceso, la accesibilidad del docente, la 
flexibilidad en el trabajo y la vinculación de los aprendizajes con el ámbito profesional.

\subsubsection{Cualitativos}

La extracción de información cualitativa de la investigación se realizó a través de una entrevista semi-estructurada a los cuatro docentes participantes en los dos grupos generados. El objetivo era conocer los pensamientos y las sensaciones de los entrevistados para obtener una perspectiva interna de la experiencia (Patton, 2002). En base a esta idea, se desarrolló un guión tomando como referencia los conocimientos previos de los investigadores y trabajos similares de investigación. Como la naturaleza de la entrevista era semi-estructurada, los investigadores pudieron añadir nuevas preguntas en base a las respuestas de los entrevistados para convertir la entrevista en una conversación (Patton, 2002). Este formato abierto permite explorar nuevas áreas para producir datos más ricos (Smith \& Osborn, 2003). Atendiendo a las variables del estudio se realizaron seis cuestiones principales a cada uno de los docentes al finalizar las asignaturas: 1) ¿Qué relevancia crees que tiene el sistema de evaluación empleado en el aprendizaje del alumno?; 2) ¿Consideras que tiene repercusión en su motivación hacia el aprendizaje?; 3) ¿Es importante permitir que el alumno se autoevalúe y coevalúe a los demás a lo largo del proceso?; 4) ¿Tiene importancia la metodología empleada para fomentar la toma de decisiones del alumno?; 5) ¿De qué manera favoreces la adquisición de autonomía del alumno en su aprendizaje?; 6) ¿Existe feedback y diálogo a lo largo del proceso para que el alumno vaya mejorando los trabajos demandados?

Toda la información relativa a estas respuestas se ha agrupado en tres categorías para su posterior análisis: 1- "Importancia del sistema de evaluación empleado", 2"Relación entre metodología y competencias docentes demostradas", 3"Transferibilidad del aprendizaje".

\subsection{Diseño y procedimiento}

Las cinco asignaturas que componen la muestra tienen un carácter semestral. El estudio queda estructurado en un diseño pretest-postest, respondiendo cada uno de los grupos a los dos cuestionarios utilizados antes y después de haber cursado las asignaturas. Todos 
los estudiantes respondieron de forma individual en el aula a cada uno de los cuestionarios. En todo momento se garantizó el anonimato para que las respuestas de los estudiantes fueran lo más sinceras posibles, al igual que se garantizó la confidencialidad en el tratamiento de los datos. Así mismo, al finalizar las asignaturas se realizó la entrevista con cada uno de los cuatro docentes participantes.

A continuación se detallan las características que tienen cada uno de los sistemas de evaluación empleados en los dos grupos generados:

- Grupo A, implicación en la evaluación: se ha utilizado un sistema de evaluación formativa basado en autoevaluaciones grupales y coevaluaciones intra e intergrupales a lo largo del proceso, regulando y registrando las horas de trabajo invertidas por los integrantes del grupo. Se utiliza un diario grupal en el que se va incluyendo el trabajo semanal realizado y el reparto de tareas entre cada componente.

- Grupo B, evaluación calificadora: no existe feedback durante el proceso y la evaluación se articula a partir de la calificación de procedimientos finales, en este caso examen y trabajos. No existen instrumentos evaluativos para la regulación del trabajo ni un proceso formativo de feedback con el docente para el asesoramiento de las tareas.

Es preciso destacar que independientemente de que el alumnado forme parte de diferentes asignaturas, titulaciones e incluso universidades, el tipo de evaluación empleada en cada uno de los grupos responde a criterios estandarizados y delimitados de antemano. Los dos docentes que imparten evaluación formativa forman parte de la Red Nacional de Evaluación Formativa y Compartida de España, aplicando de manera consensuada experiencias prácticas anuales vinculadas a este tipo de evaluación. Por lo tanto, y atendiendo tanto a la formación del profesorado como a las guías docentes, las tres asignaturas del grado de infantil se estructuran a partir de una evaluación claramente calificadora, algo que el alumno conoce y puede contrastar con su experiencia.

\subsection{Análisis empleado}


La metodología de la investigación ha sido mixta, empleándose tanto un análisis cuantitativo (descriptivo e inferencial) como cualitativo (entrevistas a docentes).

Con el diseño pretest-postest se comprueba en qué medida varía la percepción del alumno sobre su aprendizaje tras haber cursado las asignaturas, contrastando esta con la valoración que hacen los docentes que han impartido las asignaturas. La parte cualitativa del estudio se centra en el análisis de la valoración que hacen los docentes de cada grupo sobre la relevancia que tiene el uso de las estrategias metodológicas empleadas, lo que favorece el análisis de las percepciones de los alumnos. Esta complementariedad en el tratamiento de los datos otorga una visión más global de los resultados obtenidos, así como una mayor comprensión de los mismos. Además favorece la transformación de los procesos educativos desarrollados (Hall \& Ryan, 2011).

\subsubsection{Cuantitativo}

Se analizan las diferencias en las medias de las respuestas tanto en el pretest como en el postest en cada uno de los grupos, comprobando si estas son significativas tanto entre factores como antes y después del desarrollo de las asignaturas. En las correlaciones de Pearson se mide el grado de significatividad existente entre factores para cada uno de los grupos. En la ANOVA se analiza la influencia de las variables independientes de edad, expediente académico y experiencias en evaluación formativa en la transferencia del aprendizaje obtenido. Es preciso aclarar que se han analizado la distribución de las observaciones por niveles, comprobando cómo no existen problemas de varianza constante que alteren el supuesto de normalidad. Del mismo modo, también se cumple el supuesto de independencia entre las variables utilizadas. Para analizar los datos se utiliza el paquete estadístico SPSS.

\subsubsection{Cualitativo}

El análisis cualitativo se realizó a partir de la recogida estructurada de información mediante una entrevista a los docentes participantes en la investigación. Los datos extraídos se analizaron a través del análisis de la temática del contenido (Libarkin y Kurdziel, 2002) y la comparación constante entre los datos (Denzin y Lincoln, 1994). El análisis del contenido se centró en la búsqueda de patrones en el texto, codificando los 
extractos coincidentes con los patrones cruzados (Saldaña, 2009). Los temas que surgieron en el primer análisis independiente fueron examinados críticamente por todos los investigadores a través de un diálogo reflexivo. La confiabilidad fue apoyada a través de la retroalimentación continua y el análisis participativo por parte de los investigadores que revisaron y perfeccionaron las categorías emergentes, para que los resultados pudieran ser considerados confiables, creíbles y transferibles (Lincoln y Guba, 1985). El objetivo era utilizar la información obtenida para dotarla de una mayor comprensibilidad a través de la transferencia de los resultados. Las categorías que surgieron de los datos se presentan de forma explícita a través de la sección de resultados y con el apoyo de varios ejemplos de textos (Cohn, 1991).

Para recapitular, organizar y obtener la saturación de los datos a partir de las categorías generadas en las preguntas realizadas a los dos docentes se ha utilizado el programa de computación WEFT QDA. Los acrónimos empleados para los docentes del grupo A que utilizaron el sistema de evaluación formativa son DEFB (Docente Evaluación Formativa Burgos) y DEFL (Docente Evaluación Formativa León). Los utilizados para los dos que utilizaron la evaluación orientada a la calificación son DECB (Docente Evaluación Calificadora Burgos) y DECL (Docente Evaluación Calificadora León). Las categorías utilizadas se generan a partir de los objetivos del estudio. Los procedimientos de análisis que se han empleado se ciñen a las propuestas de fragmentación y articulación de la «Grounded Theory» (Strauss \& Corbin, 2002). Se realizó una codificación abierta, con categorías emergentes; codificación axial, en torno a la agrupación de las categorías emergentes en familias de subcategorías; y codificación selectiva, mediante un trabajo específico que condujo a la elaboración de un modelo interpretativo. En cada una de las entrevistas se identifica cada extracto de texto con una de las categorías delimitadas, lo que favorece la obtención de la saturación de la información a partir de la selección, recopilación y contabilización de cada uno de los fragmentos.

\section{Resultados}

\subsection{Análisis descriptivos}


Tabla 2.

Comparación de medias por factores para cada uno de los grupos en el pretest-postest (nivel de significación en las diferencias: * $p<.05$ )

\begin{tabular}{lccccccc}
\hline & \multicolumn{9}{c}{ PRE-TEST } & \multicolumn{1}{c}{ POST-TEST } \\
\hline & N & Media & DT & Var. & Media & DT & Var. \\
\hline & Grupo implicación evaluación (a) & & & \\
\hline F.1. Aprendizaje obtenido & 235 & 3.24 & .212 & .044 & 4.14 & .125 & .015 \\
\hline F.2.Competencias docentes & 235 & 3.38 & .233 & .054 & $* 4.53^{\text {aa }}$ & .107 & .011 \\
\hline & \multicolumn{6}{l}{ Grupo evaluación calificadora (b) } \\
\hline F.1. Aprendizaje obtenido & 250 & 3.43 & .196 & .038 & 3.24 & .238 & .058 \\
\hline F.2. Competencias docentes & 250 & 3.18 & .344 & .118 & $* 3.04^{\text {ba }}$ & .205 & .042 \\
\hline
\end{tabular}

*Superíndices distintos entre grupos señalan diferencias significativas a nivel .05

Tal y como se observa en la tabla 2 en el pretest no existen diferencias significativas entre factores, teniendo cada grupo unas medias similares. Sin embargo en el postest los valores del grupo A aumentan en los dos factores, existiendo diferencias respecto a antes de la asignatura en el dos, relativo a las competencias docentes. En este factor también se encuentran diferencias entre los dos grupos.

\subsection{Análisis inferencial: correlaciones de Pearson}

Tabla 3

Correlaciones de Pearson entre factores para cada uno de los grupos en el postest. $(p<.05)$

*Media F1 4.13, Media F2 4.51

\begin{tabular}{lllll}
\hline Correlaciones entre factores & $\mathrm{N}$ & Corr. de Pearson & Sig. (2-tailed) \\
\hline Grupo implicación evaluación (a) & & & \\
\hline $\begin{array}{l}\text { Factor 1. Aprendizaje obtenido/Factor } \\
\text { Competencias docentes* }\end{array}$ & 2.235 & .123 & $\mathbf{0 3 8}$ \\
\hline Grupo evaluación calificadora (b) & & & & \\
\hline $\begin{array}{l}\text { Factor 1. Aprendizaje obtenido/Factor } \\
\text { Competencias docentes }\end{array}$ & 2. & 250 & .128 & .219 \\
\hline
\end{tabular}

En la tabla 3 se refleja cómo se obtiene una correlación significativa en el

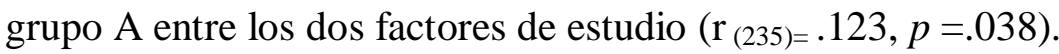

\subsection{Análisis inferencial: ANOVA}

A partir del análisis factorial realizado y en relación a las respuestas de cada uno de los grupos en los ítems relacionados con la aplicabilidad de los aprendizajes, se ha creado la variable de escala denominada "transferencia del aprendizaje". Esta variable dependiente se relaciona con otras independientes. La primera de ellas es la edad; 1 “menos de 21", 2- “entre 21 y 23”, 3- "más de 23". La segunda es la experiencia en evaluación formativa; 1- "ninguna experiencia", 2- "entre una y dos experiencias", 3"más de dos experiencias". La última variable independiente es el expediente académico; 1- "ninguna asignatura suspensa", 2- "una asignatura suspensa", 3- "más de 
una asignatura suspensa". Se realizó un ANOVA Bonferroni de un factor para grupos independientes (tabla 4). Además se realiza un post hoc indicando en qué grupos se encuentra esa diferencia significativa.

Tabla 4.

ANOVA realizado para cada una de las variables independientes analizadas en el postest.

\begin{tabular}{llll}
\hline TRANSFERENCIA DEL APRENDIZAJE & $F$ & $g l$ & $p$ \\
\hline \multicolumn{1}{c}{ Grupo implicación evaluación (a) } & & & \\
\hline Edad & 108.12 & 1 & .123 \\
\hline Experiencia previa evaluación formativa & 94.22 & 2 &. $\mathbf{0 2 3}$ \\
\hline Expediente académico & 76.17 & 1 & .143 \\
\hline Grupo evaluación calificadora (b) & & & \\
\hline Edad & 96.23 & 1 & .231 \\
\hline Experiencia previa evaluación formativa & 92.47 & 2 & .315 \\
\hline Expediente académico & 77.79 & 1 & $\mathbf{. 0 3 5 ^ { * * }}$ \\
\hline
\end{tabular}

*p< 05 entre "ninguna experiencia" (media 3.41) y "más de dos experiencias" (media 4.42)

$* * p<.05$ entre "ninguna asignatura suspensa" (media 3.13) y "más de una asignatura suspensa" (media 4.11).

Dentro del grupo que recibió una evaluación formativa las diferencias significativas se encuentran en el número de experiencias previas en estos sistemas de evaluación $\left(\mathrm{F}_{(235)}=94.22, p=.023\right)$. Los análisis post hoc, realizados mediante el procedimiento de Bonferroni han mostrado que las diferencias significativas se daban entre el grupo que no había tenido ninguna experiencia en evaluación formativa frente a los que habían tenido más de dos experiencias $(p<.05)$. De esta manera los estudiantes que han tenido más de una experiencia a lo largo de la carrera son los que perciben mayor transferencia del aprendizaje. Sin embargo, en el grupo cuya evaluación estuvo orientada a la calificación las diferencias se obtuvieron en la variable independiente del expediente académico $\left(\mathrm{F}_{(250)}=77.79, p=.035\right)$. En este caso, los análisis post hoc mostraron que las diferencias significativas $(p<.05)$ fueron entre el grupo que no tenía ninguna asignatura suspensa y los que tenían más de una asignatura suspensa. De esta manera fueron los alumnos que peor expediente tenían los que entendían este proceso evaluativo como transmisor de mayor aplicabilidad en el aprendizaje generado.

\subsection{Análisis cualitativo}

Los resultados se exponen a partir de las tres categorías de estudio generadas.

Importancia del sistema de evaluación empleado (131 extractos de texto). Se observa cómo los docentes tienen una percepción diferente sobre el rol que desempeña el tipo de 
evaluación aplicada en la asignatura. Para los del grupo A se considera clave para el aprendizaje del alumno mientras para el del grupo B es algo irrelevante:

"La evaluación empleada en la enseñanza determina la manera en la que enseñas, y más cuando se trata de futuros maestros [...]". "Es necesario prestar atención a este aspecto (no suele hacerse), ya que de cómo lo utilices depende la implicación y la motivación del alumno hacia el aprendizaje" (DEFB). "No hay que pasar por alto que los alumnos a los que enseñamos van a evaluar constantemente en su día a día $[\ldots]$ si no les damos estrategias y recursos tendrán carencias en el futuro" (DEFL).

"He evaluado toda la vida de la misma manera y la verdad no pienso cambiar a estas alturas [...]". "Yo enseño, los alumnos han de aprender y después tienen que acreditar que lo han hecho [...]". "A veces se nos olvida que estamos en la universidad". (DECL). "No creo que sea tan relevante el tipo de evaluación que se utilice, al final hay que enseñar unos contenidos y el alumno ha de adquirirlos (DECL).

Relación entre metodología y competencias docentes demostradas (122 extractos de texto). La percepción que tienen los docentes en la categoría anterior tiene una incidencia directa en la metodología desarrollada y las competencias docentes desarrolladas. Los docentes del grupo A interrelacionan la metodología utilizada con la valoración que puedan hacer los alumnos sobre sus competencias docentes. Los profesores del grupo B no le dan mucha relevancia a la valoración que puedan hacer sus alumnos sobre este hecho.

"Un profesor ha de tener unas competencias docentes elevadas [...]". "Esto no ha de ser solamente valorado por uno mismo, sino por el principal agente de aprendizaje, el alumno. "La metodología, en parte, define cómo eres como profesor, cómo te relacionas con los alumnos, la coherencia que otorgas al proceso... (DEFL). “QQuién mejor para valorar tus competencias docentes que un futuro maestro al que has formado?" (DEFB).

"Más que la metodología el principal criterio que utiliza el alumno para valorar la asignatura es la nota que saque [...] (DECB)". "En asignaturas como la mía no hay muchas posibilidades para la metodología. Tiene que ser práctica, que el alumno vivencie [...]” (DECL)

Transferibilidad del aprendizaje (119 extractos de texto). En esta categoría las valoraciones que realizan los dos profesores tienen una tendencia más similar, manifestando que es importante que el aprendizaje que adquieran los alumnos en las asignaturas pueda ser de utilidad fuera del aula:

"Cada vez hay que ser más conscientes de que la importancia no se encuentra en la cantidad de información que abarca una guía docente, sino cómo el alumno trabaja competencias en relación a los contenidos impartidos y el uso transversal que puede hacer de los mismos" (DEFB). "Es fundamental 
que cada profesor reflexione sobre la utilidad que tienen su asignatura en el desempeño profesional" (DEFL).

"Yo enseño psicomotricidad y formo a futuros maestros para que el día de mañana sean capaces de estimular el desarrollo psicoevolutivo del niño [...]". "A veces puede gustar más o menos a los alumnos, pero intento enseñar todo aquello que considero necesario" (DECB). "Cada vez la forma de enseñar debería ser más práctica, dando recursos al alumno que pueda aplicar en el aula” (DEFB).

\section{Discusión}

Se ha demostrado en la presente investigación la relevancia que tiene el sistema de evaluación empleado en la percepción del alumnado sobre su aprendizaje y las competencias desarrolladas por el docente. El grupo A, al que se le permitió ser partícipe de su propio proceso de evaluación mediante autoevaluaciones grupales y coevaluaciones intra e intergrupales, valoró tras finalizar la asignatura un mayor nivel de competencias docentes puestas en práctica. En ambos grupos la percepción difiere en función de las variables independientes de experiencias previas en evaluación formativa y expediente académico. Por otro lado, las valoraciones que hacen los dos docentes sobre el rol que desempeña la metodología y la evaluación difieren sustancialmente.

Es destacable que antes de impartirse las asignaturas no se obtuvieron diferencias significativas entre grupos en ninguno de los dos factores, lo que refleja la similitud de percepciones de los estudiantes. Esto es debido, en parte, a que en años anteriores han recibido sistemas de evaluación diferentes en las asignaturas cursadas, al no existir un planteamiento consensuado entre los docentes sobre el método a utilizar. En este sentido, experiencias como las de Elton (2010) indican que cuando el alumno se acostumbra a una manera de trabajar, independientemente de cuáles sean los contenidos abordados, se favorece la continuidad y la sistematicidad entre asignaturas. Esto deriva en que no tenga que exponerse a un sistema de evaluación específico para cada una, en muchos casos demandando la realización de procedimientos de evaluación similares pero con procesos de enseñanza totalmente diferentes.

Otro aspecto destacable es que las valoraciones previas entre grupos sean semejantes. Es un aspecto positivo en diseños metodológicos pretest-postest, ya que indica que los resultados obtenidos tras la intervención realizada serán consecuencia de la misma, y por lo tanto más significativos (Garaigordobil, 2007). Es en el postest donde 
se encuentran las diferencias entre grupos, sobre todo en el factor de competencias docentes. Éstas son significativamente más elevadas para el grupo que se implicó en su propia evaluación, lo que refleja una visión más positiva del alumno hacia el docente cuando éste realiza planteamientos metodológicos más abiertos y flexibles. Además, en este caso en el que se forma a futuros docentes tanto de la etapa de primaria como de infantil, existe una vinculación directa entre el grado de satisfacción del alumno en las estrategias de enseñanza vivenciadas y los modelos pedagógicos que utilizará cuando sea docente (Meyer y Murrell, 2014). En el factor dos, dentro del grupo A, también se incrementan significativamente los valores en el postest respecto al comienzo de la asignatura, lo que demuestra de nuevo el cambio manifestado dentro del grupo, quizás acostumbrado en asignaturas anteriores a otros procesos evaluativos.

Es preciso destacar que una vez finalizan las asignaturas las medias de los dos factores descienden en el grupo que recibió una evaluación orientada a la calificación. Este hecho manifiesta el descontento de los alumnos con el planteamiento metodológico y evaluativo recibido, incidiendo en una percepción más baja sobre el aprendizaje adquirido. Hortigüela, Pérez-Pueyo y Abella (2015) indican que las metodologías de carácter tradicional, fundamentadas en la exposición de temas por parte del docente y la participación secundaria del alumnado en la gestión de su proceso de aprendizaje, derivan en una menor implicación del alumno y como consecuencia en una menor inversión de horas para la realización de las tareas.

Por otro lado se observa cómo al finalizar el proceso existe una correlación significativa entre los dos factores de estudio en el grupo A. Los alumnos que valoraron de manera elevada el aprendizaje adquirido también lo hicieron sobre las competencias docentes utilizadas por el profesor. Se demuestra que variables como la planificación de las tareas, la interacción con el alumnado y la flexibilidad en los planteamientos didácticos son valorados positivamente por el alumnado para la adquisición de conocimientos y destrezas (Alonzo, Kobarg y Seidel, 2012).

En relación a la variable dependiente de la transferencia del aprendizaje adquirido, entre los alumnos que recibieron la evaluación formativa las diferencias se encontraron en la variable de experiencias previas en estos sistemas. Fueron aquellos que más veces han vivenciado estos procesos los que consideran una mayor 
transferibilidad. Esto denota lo favorable que es que el alumnado se habitúe a estos sistemas así como a los problemas iniciales que se pueden generar en un primer momento cuando el alumno no está acostumbrado (Fraile, 2012).

En el grupo que recibió el sistema de evaluación orientado a la calificación las diferencias surgieron en la variable del expediente académico. Los alumnos que menos valoraron la transferencia fueron los que tenían menos asignaturas suspensas. Se asocia por lo tanto un mejor expediente académico a la valoración negativa de sistemas de evaluación orientados a la calificación. Esto puede relacionarse con estudios como el de López-Pastor y Palacios (2012) que determinan que cuando el alumnado obtiene resultados positivos sin exigirle mucho trabajo se muestra conforme con el proceso desarrollado y reacio al cambio.

En la parte cualitativa del estudio se ha observado cómo los docentes participantes tienen una percepción muy dispar sobre el rol que desempeña la evaluación en el proceso formativo, repercutiendo en el uso de estrategias metodológicas con fines contrapuestos. En relación al sistema de evaluación empleado el docente del grupo A otorga mucha importancia a la manera en la que se aborda y se implementa en las asignaturas, relacionando su uso con la implicación de los alumnos en el proceso. Sin embargo, el profesor del grupo B no considera que haya que prestar mucha atención a la innovación en la evaluación. Molina, Valencia-Peris \& Suárez (en prensa) indican que es fundamental reflexionar acerca de la manera en la que gestionamos los procesos educativos, ya que actualmente no es tan importante el volumen de información que se enseña como la herramientas aplicadas para adquirirlo.

Respecto a su percepción sobre el uso metodológico y las competencias docentes desarrolladas, de nuevo el docente del grupo B no reflexiona sobre la adecuación de la misma a las asignaturas; destacando que el alumno valora la asignatura de manera positiva si su calificación ha sido elevada. Por el contrario, el profesor del grupo A da importancia al hecho de lo que pueden opinar los alumnos sobre las competencias docentes puestas en práctica, más tratándose de futuros docentes. Experiencias como las de Espinosa (2013) indican que cuando el alumno ha pasado por procesos de evaluación formativa, y se han definido los criterios de calificación de 
manera clara desde el comienzo, reconoce el sistema como mayor generador de aprendizaje aunque su nota no sea demasiado elevada.

Finalmente en la tercera categoría, relativa a la transferencia del aprendizaje, los dos docentes reflejan un mayor grado de acuerdo sobre la practicidad y utilidad que han de tener los conocimientos que enseñamos. Hortigüela, Pérez-Pueyo y Hernando (2015) reflejan la relevancia que tiene este hecho, siendo además algo que los estudiantes valoran de una manera elevada.

\section{Conclusiones}

En relación al primer objetivo, se ha observado cómo el sistema de evaluación empleado ha tenido una incidencia significativa en la percepción del alumnado, tanto en su aprendizaje como en las competencias desarrolladas por el docente. Es en estas competencias donde se establecieron diferencias significativas favorables para el grupo que recibió la evaluación formativa.

Respecto al segundo objetivo, los alumnos del grupo A que más veces han participado en procesos de evaluación formativa perciben una mayor transferibilidad en los aprendizajes. Sin embargo en el grupo B esta transferencia se ve influenciada por el expediente académico.

Como respuesta al tercer objetivo, se han obtenido percepciones muy diferentes entre los dos docentes en relación al rol que desempeña la evaluación en el proceso de enseñanza y el empleo de metodologías vinculadas a las competencias docentes. El profesor del grupo A valora mucho más estas dos variables, adecuándolas en el aula en función de aquello que enseña y la manera en la que lo hace. Sobre la transferibilidad de los aprendizajes los dos profesores manifiestan un mayor acuerdo.

Consideramos que este artículo puede ser de especial interés para aquellos docentes, de cualquier área de conocimiento, interesados en el diseño y aplicación de sistemas de evaluación y planteamientos metodológicos abiertos y participativos. También para los Vicerrectores de Ordenación Académica y/o coordinadores de elaboración de actividades formativas para el profesorado universitario, ya que a través de la propuesta de cursos o seminarios se permite al docente reflexionar sobre su práctica y reajustar sus guías docentes. El estudio presenta algunas limitaciones. En 
primer lugar únicamente se atiende a alumnado de una misma facultad, por lo que sería interesante contrastar estos resultados con estudiantes de otras titulaciones. En segundo lugar, la intervención se lleva a cabo solamente a lo largo de un semestre. Podría ampliarse este estudio mediante un análisis más longitudinal, comprobando el efecto que produce en el alumnado la aplicación de estos sistemas a largo plazo.

\section{Referencias bibliográficas}

Alonzo, A., Kobarg, M. \& Seidel, T. (2012). Pedagogical Content Knowledge as Reflected in Teacher-Student Interactions: Analysis of Two Video Cases. Journal of Research in Science Teaching, 49, 10, 1211-1239.

Castejón, F. J., Santos, M. y Palacios, A. (2015). Cuestionario sobre metodología y evaluación en formación inicial en Educación Física. Revista Internacional de Medicina y Ciencias de la Actividad Física y el Deporte, 15,58, 245-267.

Chan, C. (2015). Tensions and Complexities in School-University Collaboration. Asia Pacific Journal of Education, 35(1), 111-124.

Canabal, C. y Castro, B. (2012). La evaluación formativa ¿la utopía de la educación superior? Pulso: Revista de Educación, 35, 215-229.

Cohn, P. J. (1991). An exploratory study on peak performance in golf. The Sport Psychologist, 5, 1-14.

Corbetta, P. (2007). Metodologías y técnicas de investigación social. Madrid: McGraw Hill.

Dekker-Groen, A., Van der Schaaf, M. \& Stokking, K. (2013). A Teacher Competence Development Programme for Supporting Students' Reflection Skills. Teachers and Teaching: Theory and Practice, 19, 2, 150-171.

Denzin, N. K. \& Lincoln, Y. S. (1994). Handbook of Qualitative Research. Thousand Oaks, CA: Sage.

Elton, L. (2010). Complexity Theory: An Approach to Assessment that Can Enhance Learning and More Generall y Could Transform University Management. Assessment \& Evaluation in Higher Education, 35,5, 637-646. 
Espinosa, M.T. (2013). Evaluación de competencias mediante rúbrica. Importancia de las matemáticas en la evaluación de competencias genéricas. Historia y Comunicación Social, 18, 2, 243-255.

Fraile, A. (2012). Evaluación formativa e interdisciplinariedad. Análisis de dos asignaturas con el mismo sistema de evaluación. Psychology, Society \& Education, 4,1, 5-16.

Garaigordobil, M. (2007). Perspectivas metodológicas en la medición de los efectos de un programa de intervención con adolescentes. La evaluación pretest-postest y los cuestionarios de evaluación del programa. Apuntes de Psicología, 25, 3, 357-376.

Gikandi, J. (2013). Synergy between Authentic Assessment Activities and Learner Autonomy: How Does This Promote Shared Authenticity in Online Higher Education? International Journal on E-Learning, 12, 4, 353-381.

Hall, J. \& Ryan, K. (2011). Educational Accountability: A Qualitatively Driven MixedMethods Approach. Qualitative Inquiry, 17, 1, 105-115.

Heyvaert, M., Hannes, K., Maes, B. \& Onghena, P. (2013). Critical Appraisal of Mixed Methods Studies. Journal of Mixed Methods Research, 7, 4, 302-327.

Hidalgo, A., Bordallo, J., Sánchez, M., y Cantabrana, B. (2012). Protagonismo de los alumnos en el aprendizaje. Una experiencia en el primer curso de medicina. FEM. Revista de la Fundación Educación Médica, 15, 4, 213-219.

Hortigüela, D., Pérez Pueyo, A. y Abella, V. (2015). Perspectiva del alumnado sobre la evaluación tradicional y la evaluación formativa. Contraste de grupos en las mismas asignaturas. REICE. Revista Iberoamericana sobre Calidad, Eficacia y Cambio en Educación, 13, 1, 35-48.

Hortigüela, D., Pérez-Pueyo, A., y López-Pastor, V.M. (2015). Implicación y regulación del trabajo del alumnado en los sistemas de evaluación formativa en educación superior. Relieve: Revista Electrónica de Investigación y Evaluación Educativa, $21,1,1-5$. 
Hortigüela, D., Pérez-Pueyo, A. y Hernando, A. (2015). Relación entre el aprendizaje generado en el aula y la motivación del alumnado hacia el desempeño profesional. Revista de Investigación y Estudios Gerenciales,19, 59-69.

Johnson, (2014). Healthcare Learning Community and Student Retention. InSight: A Journal of Scholarly Teaching, 9, 3, 28-35.

Libarkin, J. C. \& Kurdziel. J. P. (2002). Research methodologies in science education: Qualitative data. Journal of Geoscience Education, 50, 195-200.

Lincoln, Y. S. \& Guba, E. (1985). Naturalistic inquiry. Newbury Park, CA: Sage.

López-Pastor, V. M. y Palacios, A. (2012). Percepción de los futuros docentes sobre los sistemas de evaluación de sus aprendizajes. Teoría de la Educación: Educación y Cultura en la Sociedad de la Información, 13(3), 317-341.

Meyer, K., \& Murrell, V. (2014). National Survey of Faculty Development Evaluation Outcome Measures and Procedures. Online Learning 18, 3, 16-29.

Molina, J. P., Valencia-Peris, A. y Suárez, C. (en prensa). Percepción de los estudiantes de una experiencia de uso didáctico de blog docente en Educación Superior. Educación XX1.

Moreno-Murcia, J. A., Silveira, Y. y Belando, N. (2015). Cuestionario de evaluación de las competencias docentes en el ámbito universitario. Evaluación de las competencias docentes en la universidad. New Approaches in Educational Research, 4, 1, 54-61.

Patton, M. Q. (2002). Qualitative Research and Evaluation Methods. Thousand Oaks, CA: Sage.

Peiró, S. (2012). Innovaciones sobre la formación inicial de profesores con relación a la educación en valores. Revista Electrónica Interuniversitaria de Formación del Profesorado, 15, 1, 61-80.

Saldaña, J. (2009). The Coding Manual for Qualitative Researchers. Thousand Oaks, CA: Sage.

Serván, M.J. (2011). Percepciones e interpretaciones de las comunidades educativas en torno a la evaluación externa de los aprendizajes escolares. Estudios de caso 
sobre la evaluación de diagnóstico en Andalucía. Cultura y Educación: Revista de Teoría, Investigación y Práctica, 23, 2, 221-234.

Smith, J. A. y Osborne, M. (2003). Interpretative Phenomenological Analysis. En J. A. Smith (Ed.) Qualitative psychology: A practical guide to research methods. (pp. 53-79). London: Sage.

Strauss, A. \& Corbin, J. (2002). Bases de la investigación cualitativa: Técnicas y procedimientos para desarrollar la teoría fundamentada. Colombia: Universidad de Antioquia.

Varsavsky, C., Matthews, K. \& Hodgson, Y. (2014). Perceptions of Science Graduating Students on Their Learning Gains. International Journal of Science Education, $36,6,929-951$.

Wold, K. (2013). Collaborative Inquiry: Expert Analysis of Blended Learning in Higher Education. International Journal on E-Learning, 12, 2, 221-238. 